Results At least five stand-alone surveillance systems operated by different agencies in the West Bank collect data related to road traffic accidents. These systems do not have a common set of indicators, no formal case definitions or standard operating procedures (SOPs). There is a lack of quality assurance systems at all levels. There are large discrepancies in reported casualties: for 2012 the Palestinian Civil Police (PCP) reported 120 fatalities, whereas $\mathrm{MoH}$ reported both 34 and 112 from separate sources of data. Privacy concerns hinder some organisations from sharing data. $\mathrm{MoH}$ and PCP data are sufficiently complete to support a robust integrated registry.

Conclusions An integrated registry for RTC is feasible. The establishment should comprise a framework for all essential parts of a registry, including stakeholder relations and a comprehensive quality assurance system. Steps toward technical improvement include establishing a multisectoral working group; developing a common set of indicators and case definitions; revising data collection forms and developing SOPs for the whole continuum of data flow.

\section{FACTORS CAUSING DEATHS DUE TO INJURY AMONG CHILDREN IN BANGLADESH}

${ }^{1}$ Md Hasinur Rahaman Khan, ${ }^{2}$ Tahera Ahmed, ${ }^{3,4}$ Faisal Ababneh. ${ }^{1}$ Applied Statistics, Institute of Statistical Research and Training, University of Dhaka, Bangladesh; ${ }^{2}$ Centre for Injury Prevention and Research, Bangladesh; ${ }^{3}$ Department of Mathematics and Statistics, Sultan Qaboos University, Oman; ${ }^{4}$ Department of Mathematics, Al-Hussein Bin Talal University, Maan, Jordan

\subsection{6/injuryprev-2016-042156.730}

Background Child injury has become a public health concern in both developing and developed world. In recent times, injury has been identified as a leading cause for both morbidity and mortality among children. This study has concentrated on a holistic statistical inquest into injuries and deaths among children in Bangladesh.

Methods A population-based cross-sectional survey was conducted between January and December 2003 in Bangladesh. Nationally representative data were collected from 171,366 rural and urban households, with a total sample size of 819,429 . A simple association test and the binary logistic regression was designed to identify the factors causing child death due to injury. Results Drowning found the leading cause of death of children in Bangladesh. Approximately 26\% children died from drowning each year. Almost $50 \%$ of children died in a year from just because they did not get proper care after getting injured. Mother's education has a significant influence on child injury and death. The percentage of child death due to injury was the highest from a mother's lack of awareness and education (54\%). The odds of dying due to injury among children of secondary and graduate level educated mothers are respectively 1.4 and 1.6 times more than the odds in the chances of death of an illiterate mother's child. This may be due to the fact that educated mothers have less time to supervise their children because of their daily activities. It was also found that almost $63 \%$ of the children who died from injury were from rural areas.

Conclusion Child injury is an emerging cause of mortality and morbidity in both urban and rural areas of Bangladesh. Children from rural area were the more vulnerable group for different types of injuries compared to urban population. The home is the most common place for injury occurrence.

Acknowledgements The financial support of UNICEF is acknowledged.

\section{POTENTIAL UNDERESTIMATION OF ROAD TRAFFIC INJURY MORTALITY IN MEXICO: SUBNATIONAL ANALYSIS}

${ }^{1}$ Ricardo Pérez-Núñez, ${ }^{2}$ Mariana G Mojarro-lñiguez, ${ }^{1}$ Ma Eulalia Mendoza-García, ${ }^{1}$ Sergio Rodrigo Rosas-Osuna, 'Martha Hijar-Medina. ' ${ }^{1}$ Secretariado Técnico Del Consejo Nacional Para La Prevención De Accidentes, Secretaría De Salud (National Council for Accident Prevention Secretariat, Ministry of Health); ${ }^{2}$ Independent Researcher

\subsection{6/injuryprev-2016-042156.731}

Background Quality of data is essential to best understand the real magnitude and consequences of road traffic injuries (RTI). The objective of this study was to estimate the potential underestimation of RTI mortality at the subnational level in Mexico for a period of 15 years and to identify social and economic variables at the state level potentially associated with the quality of statistical classification of deaths.

Methods We conducted secondary analysis of validated mortality databases for the period 1999-2013. Five categories of relevant "garbage codes" pertinent for RTI were identified and the percentage they represent of the total was estimated. Using multiple imputation models, registries statistically likely to be due to RTI were estimated and the potential underestimation of mortality was quantified. We explore correlation between health resource availability, social and economic variables with the percentage of underestimation of mortality caused by RTI at the state level using the Kendall's rank correlation test.

Results $1.99 \%$ of all deaths were assigned to "R" codes; $2.40 \%$ were injuries of undetermined intent; in $22.96 \%$ of unintentional injuries the external cause is not codified; and $0.11 \%$ of transport injuries did not specify the means of transport. In over $40 \%$ of RTI, the specific road user deceased was unknown. The potential underestimation of deaths from RTI during the period was $18.85 \%$ at the national level, with significant variations amongst the 32 Mexican states, varying from $5.32 \%$ in Queretaro to $51.49 \%$ in Baja California. From the data analysed, there was no statistical evidence of any association of the percentage of RT deaths underestimation with variables analysed.

Conclusions Performance in terms of mortality classification is different at the state level, but more analysis is needed to better understand underlying reasons of garbage coding. This information is useful for targeting interventions to improve recording of deaths in Mexico.

\section{FALLS - ONLY A HEALTH RISK FOR THE ELDERLY? RESULTS OF THE "GERMAN HEALTH UPDATE"}

Anke-Christine Sass, Alexander Rommel, Gianni Varnaccia. Robert Koch Institute, Germany

\subsection{6/injuryprev-2016-042156.732}

Background Unintentional injuries (UIs) caused by falls are an important public health issue in ageing populations. This paper describes the incidence and consequences of falls in the adult population in Germany; the focus is on age and gender differences.

Methods The representative phone survey "German Health Update 2010" ( $\mathrm{n}=22,050)$ provides information on up to three medically treated UIs within twelve months. Analyses focus on the characteristics of falls compared to other types of UIs. Frequencies and 95\% confidence intervals (CI: 95) were calculated and logistic regression was applied to control for confounders.

Results Like UIs in general, falls are more frequent in younger than in older age, particularly in men. The prevalence decreases 
from 5.3\% (CI95: 4.2-6.7) in men aged $18-29$ to $1.8 \%$ (CI95: 1.0-3.3) in men aged 70 and over. However, at the same time, the proportion of falls among all UIs rises with age: from $22.7 \%$ (CI95: 18.4-27.7) to 52.6\% (CI95: 32.7-71.7) in men and from 20.1\% (CI95:15.2-26.0) to 63.9\% (CI95: 47.7-77.5) in women. The proportion of falls that occur at home also increases significantly with age: from $14 \%$ to over 50\%. Still, among the elderly, about one third of falls occurs in public places (among pedestrians). The consequences of UIs caused by falls are often serious: 34.4\% (CI95:29.8-39.4) of all falls cause bone fractures (all UIs: 20.4\%; CI95: 18.3-22.6). One-quarter (26.8\%; CI95: 22.431.7) result in hospitalisation (all UIs: 18.3\%; CI95: 16.2-20.5). Moreover, the need for physical therapy and rehabilitation is higher after falling compared to all other UIs.

Conclusions The data demonstrate the severity of UIs caused by falls and reveal important age and gender specific patterns. Older people are no more at risk than younger people, but the consequences for them are more serious than for the younger. Consequently, fall-related UIs are of importance in all ages and require age and gender specific approaches to prevention.

\section{DEVELOPING A MODEL FOR PROCESSING THE INFORMATION OF ROAD TRAFFIC INJURIES AS THE DATABASE FOR COLLABORATIVELY PLANNING OPERATIONAL MEASURES TO REDUCE THE ROAD TRAFFIC ACCIDENTS}

'Patcharawadee Pirunsuntorn, ${ }^{2}$ Wilai Thanalad, ${ }^{1}$ Adisak Khongwattananont. ${ }^{1}$ Khonkaen Provincial Public Health Office; ${ }^{2}$ Kranuan Crown Prince Hospital

\subsection{6/injuryprev-2016-042156.733}

Background Based on the global status report on road safety in 2013 by World Health Organisation (WHO), Thailand ranked third in the list of countries having highest road traffic deaths worldwide (reported number of road traffic deaths Estimated number of road traffic deaths; Point estimate 26,312 Estimated road traffic death rate per 100000 population approximately 26,312 or at the death rate of 38 road fatalities per 100,000 inhabitants). The median value of the total number of road fatality in Khonkaen during2011-2013 was the fourth highest nationwide (468 or at the death rate of 26.49 road fatalities per 100,000 inhabitants).

Description of the problem The information from the injury surveillance and the dead case investigation recorded by Khonkaen provincial public health office have not been utilised at its maximum efficiency; less information can be accessible by the network units. Consequently, each unit has individually managed its assignment in solving the problems on road traffic accidents. Thus, to integrate the operational procedures of all the units concerned in order to create a co-operation and collaborative operational guidelines for them to follow, Khonkean provincial public health office arranged a venue for returning the information to those units in parallel with developing a model in processing the recorded information of road traffic injuries as the database available for all the network units to easily access into the system and make use of the records for collaboratively planning the operational measures serving the safety road scheme.

Results Obtained a model for managing the information processing systemby integrating the operational procedures of all the network units beginning from the regular surveillance, the case investigation, to the delivery of information to the communities and encouraged to have the findings utilised for integrated problem solving both in short term and long term; and 2) establishment of community checkpoints at the village level to reduce road traffic accidents during festival seasons.

Conclusions Obviously, social tendency of realisation alert for safety road was widely enhanced; this was the result from the understanding of the road traffic safety and the integration of problem solving procedures for road traffic accidents among all the parties concerned.

\section{FACTORS INFLUENCING SOCIAL AND HEALTH OUTCOMES AFTER LAND TRANSPORT INJURY: RECRUITMENT AND PARTICIPANT CHARACTERISTICS, SHORT TERM HEALTH AND SOCIAL STATUS}

Jagnoor Jagnoor, Ian Cameron, on behalf of the FISH study investigators. University of Sydney, Australia

\subsection{6/injuryprev-2016-042156.734}

Background There is growing evidence that health and social outcomes following motor vehicle crash injury are related to cognitive and emotional responses of the injured individual, as well as relationships between the injured individual and the compensation systems with which they interact. Investigation is therefore warranted to identify the key determinants of health and social outcomes following injury in the context of the New South Wales motor accident insurance scheme.

Methods In this inception cohort study, 2400 participants, aged 17 years or more, injured in a motor vehicle crash in New South Wales are being identified principally though hospital emergency departments. Participants will be initially contacted through mail. Baseline interviews are conducted by telephone within 28 days of the injury and participants are followed up with at 6, 12 and 24 months post-injury. Medicare and Pharmaceutical prescription data will also be linked to the observed data.

Results Recruitment for the study is underway with 1438, 924, 706, 135 baseline, 6, 12 and 24 months interviews completed respectively. Interim analysis of first 777 participants is reported below. Over one- fourth $(215 ; 27.3 \%)$ of the participants were born outside Australia, 67\% were males, $79.5 \%$ were in paid employment at the time of injury. The data source/hospital was significantly associated with the distribution of mode of transport injuries and major differences were observed for urban hospitals with $35.9 \%(232 / 647)$ of the cohort being bicyclists whilst a high proportion of motorcycle riding injuries $(51.6 \%$; 48/93) were reported from rural hospitals. At the first interview, participants had a lower health related quality of life than the general population (EQ5D a mean difference of -0.539 ; <0.0001); despite less than half reporting admission to hospital because of their injury. Return to work was reported by $65 \%$ whilst only $36 \%$ reported being able to return to their usual social activities. Analysis of outcome predictors related to post-injury function, disability and return-to-work are now under way.

Conclusions The interim analysis of the cohort reported a very high proportion of bicyclists injuries. Identification of factors associated with health and social outcomes following injury, including related compensation factors will provide evidence for improved service delivery, post-injury management, and inform policy development and reforms. 